\title{
COMPARATIVE STUDIES ON SYNTHESIS AND STRUCTURE OF OLD AND NEW POLYMORPHS OF DESVENLAFAXINE - AN ANTIDEPRESSANT DRUG
}

\author{
Mahendra Khandpekar ${ }^{1} *$, Jayanta Mheta ${ }^{2}$, Sanjay Patil ${ }^{2}$, Sonali Araj $^{2}$, Pandurang \\ Satpute ${ }^{3}$
}

\begin{abstract}
1Materials Research Lab, Department of Physics, Birla College, Kalyan - 421 304, India. 2Department of Chemistry, Changu Kana Thakur, Arts, Commerce and Science College, New Panvel, Raigad - 410 206, India

3Department of Chemistry, Birla College, Kalyan - 421 304, India.

Submitted: $18-08-2013$

Revised: 09-10-2013

Accepted: 02-12-2013

*Corresponding author

Mahendra Khandpekar

E-mail address:

dr_mmk1968@yahoo.com
\end{abstract}

\begin{abstract}
Desvenlafaxine succinate (pristiq) is an important nonhormonal drug used in the treatment of major depressive disorder and problems associated with menopause. This article summarizes the synthesis and investigation of new polymorphic form of Desmethylvenlafaxine. The new polymorph has been compared with the old polymorph using XRD, DSC, FTIR, SEM and HPLC techniques. Three prominent reflections with higher intensities lying at 20.3, 13.1 and 15.8 have been noted in the XRD measurements for the new polymorph. Higher intensity (XRD) peaks for the new polymorph indicates good crystalline quality. FTIR spectra shows close match between the polymorphs. DSC measurements show enhanced thermal stability for the new polymorph. Higher purity is indicated by the HPLC studies.

Key words: desvenlafaxine, polymorphism, structure, crystalline, purity
\end{abstract}

\section{INTRODUCTION}

Major depressive disorder is considered to be the most incapacitating conditions in the world Lourenco and Kennedy, 2009. The drug desvenlafaxine succinate $\left(\mathrm{C}_{16} \mathrm{H}_{25} \mathrm{NO}_{2}\right.$, Mol. Wt: $263.375 \mathrm{~g} / \mathrm{mol}$ ) has long been used for the treatment of the major depressive disorder (De Martinis et al., 2007; Abirami et al., 2011. Popularly known as pristiq or Odesmethylvenlafaxine, it has being targeted as the first non-hormonal drug for treatment of menopause (Umand and Falconieri 2012, Archer et al., 2009) several polymorphic forms each having distinct crystal structures and physical properties like melting point, X-ray diffraction pattern and infrared absorption fingerprint (Brittain, 2009). Moreover the crystalline forms may be distinguished on the basis of their thermal behavior different. Thermal behavior can be majored by techniques such as capillary melting point, differential scanning calorimetry (DSC), which have been used to distinguish the polymorphic forms (Giron, 2002).

In the present investigation the old as well as new polymorph of desvenlafaxine has been prepared in our laboratory and the method of preparation has been outlined. The two samples of desvenlafaxine have been compared on the basis of XRD, IR, DSC (thermal), SEM and HPLC chromatographic studies. The structure of our new polymorph is found to be in close agreement with that of desvenlafaxine succinate monohydrate (Venu, et al., 2008). The new polymorph reported in this paper is found to be more pure and thermally stable as compared to the old polymorph of desvenlafaxine as detailed below.

\section{MATERIAL AND METHODS}

All reagents, chemicals and solvents used for the synthesis were of AR grade. The XRD pattern of the samples was recorded on Shimadzu XRD 6000 with a solid state detector using $\mathrm{Cu}_{\alpha}$ radiation $\left(1.5418 \mathrm{~A}^{\circ}\right)$. The sample holder consisted of a round standard aluminum holder with a rough zero background. The sample was scanned between 2-40 (20) degrees with step size of $0.02^{\circ}$ at a rate of $2^{\circ} / \mathrm{min}$. The Infrared spectra was recorded on a FTIR infrared Shimadzu 8400 spectrophotometer in the range from 400 to $4000 \mathrm{~cm}^{-1}$. 
<smiles>N#CCc1ccc(OCc2ccccc2)cc1</smiles><smiles>OC(c1ccc(OCc2ccccc2)cc1)C1(O)CCCCC1</smiles><smiles>NCC(c1ccc(OCc2ccccc2)cc1)C1(O)CCCCC1</smiles><smiles>CN(C)CC(c1ccc(OCc2ccccc2)cc1)C1(O)CCCCC1</smiles><smiles>CN(C)CC(c1ccc(O)cc1)C(O)(CN(C)C)c1ccc(OCc2ccccc2)cc1</smiles>

Figure 1. Reaction mechanism for synthesis of new polymorph of desvenlafaxine

Perkin Elmer (DSC7) analyzer was used for Differential Scanning Calorimetry Measurements. A Vergo make melting point apparatus was used for estimation of melting point. SEM was recorded using Jeol JSM 6500F scanning electron microscope. HPLC Chromatograph was recorded on a Perkin Elmer series 200 HPLC analyzer system.

\section{Normal method of synthesis}

For a batch quantity of $10 \mathrm{~g}$, desvenlafaxine was prepared by reacting Odesmethylvenlafaxine with methanol and ethyl acetate. To begin with methanol in a fournecked round bottom flask was stirred at room temperature and desmethylvenlafaxine base was added to it. This was followed by addition of ethyl acetate to the reaction mixture again under stirring. The mixture was heated till the temperature of the reaction mass was raised to $80^{\circ} \mathrm{C}$. This temperature was maintained till complete dissolution of o-desmethylvanlafaxine occurs. The heating was then discontinued and the material was allowed to cool naturally upto $30^{\circ} \mathrm{C}$ with continuous stirring so that product can be precipitated out. The product was then separated from the reaction mass by filtering it. The final product was then washed with a small quantity of methanol and dried at $60^{\circ} \mathrm{C}$ under vacuum to get the crystalline product.

\section{Synthesis of old polymorph}

4-[2-amino-1-(1-hydroxycyclohexyl)

ethyl] phenol $(2.12 \mathrm{~g} ; 0.009 \mathrm{~mol})$ was suspended in $21.2 \mathrm{~mL}(16.79 \mathrm{~g})$ of methanol under nitrogen atmosphere. A white suspension was obtained to which $1.69 \mathrm{~mL}(0.022 \mathrm{~mol})$ of formaldehyde $(37 \%)$ was added followed by $4.46 \mathrm{~g}(0.021 \mathrm{~mol})$ of sodium triacetoxyboro-hydride in portions. The resulting suspension was then stirred for $4 \mathrm{~h}$ and $40 \mathrm{~min}$ at room temperature. Following which $21.2 \mathrm{~mL}$ Lof water was added, and the stirring was continued at room temperature for $10 \mathrm{~min}$. The methanol was then removed by distillation, and the resulting solution was washed twice with $10 \mathrm{~mL}(8.67 \mathrm{~g})$ of toluene. 
Table I. XRD reflections at $2 \theta$ degrees for old and new polymorphs of desvenlafaxine

\begin{tabular}{ccc}
\hline Obs. No & New Polymorph & Old Polymorph \\
\hline 1 & 12.0 & 12.1 \\
2 & 13.1 & 13.2 \\
3 & 15.8 & 15.9 \\
4 & 20.3 & 20.4 \\
5 & 22.3 & 22.3 \\
\hline
\end{tabular}

Thereafter, the $\mathrm{pH}$ was adjusted to 9.5 by adding $50 \%$ sodium hydroxide to it. The resulting suspension was cooled to $0-5^{\circ} \mathrm{C}$ and was filtered. The wet solid was finally dried under vacuum at $60^{\circ} \mathrm{C}$ until constant weight was obtained.

\section{Synthesis of new polymorph}

The reaction mechanism can be summarized as follows. Initially 4-benzyloxypehnyl acetonitrile and cyclohexanone was condensed in the presence of potassium tertiary butoxide as base in n-butanol to get DFL-I. The cyano group of DFL-I undergoes hydrogenation in methanolic ammonia and Raney nickel as catalyst to form DFL-IIA. DFL-IIA is then reacted with para formaldehyde and formic acid and isolated using sodium hydroxide solution to get dimethylated product DFL-III.A. DFL-IIIA was then hydrogenated with $\mathrm{Pd} / \mathrm{C}$ in methanol to get debenzylated product DFL-IV (Desvenlafaxine crude). The crude product was finally purified by charcoalisation in methanol and ethyl acetate to get final new crystalline polymorph of desvenlafaxine. The stepwise process of preparation of the new polymorph is shown in figure 1.

\section{RESULTS AND DISCUSSION XRD analysis}

To confirm the phase formation the samples in powder form were exposed to $\mathrm{X}$ ray radiation. Table I lists the prominent XRD peaks for the polymorphs. Three strong peaks each are found in both new as well as old polymorph lying near $20.4^{\circ}, 13.2^{\circ}$ and $15.8^{\circ}$. The intensity ratio of the new polymorph was found to be ten times greater than the old polymorph. The higher intensities in new polymorphs clearly indicate better phase formation. The highest intensity peak around $20.3^{\circ}$ is found to be bifurcated in the old polymorph.

\section{Thermal DSC studies}

Thermal Differential Scanning Calorimetry (DSC) studies indicate sharp melting endotherm near the melting points of the respective polymorphs. Melting point of the older polymorph lies at $225^{\circ} \mathrm{C}$ as compared to the new polymorph which was found to lie at $230^{\circ} \mathrm{C}$. The new polymorph thus has greater thermal stability then the old polymorph. The melting points were also confirmed using conventional melting point apparatus.

\section{SEM analysis}

The scanning electron microphotographs (SEM images) of the old and the new polymorphic forms are shown in figure 2 and figure 3 . The crystalline aggregates in the old polymorph are clearly transformed in to more regular, elongated needle shaped structures. The new polymorph is thus more ordered than the old polymorph exhibiting crystalline nature. Particle shape of new compound shows a remarkable change from previously existing one. The new polymorph is also found to have enhanced solubility.

\section{FTIR studies}

For identification of fundamental groups present Fourier Transform Infrared Spectral (FTIR) analysis using $\mathrm{KBr}$ pellet technique was carried out for the new polymorph. Table II shows a comparative chart of the IR peaks for old and new polymorphs. 


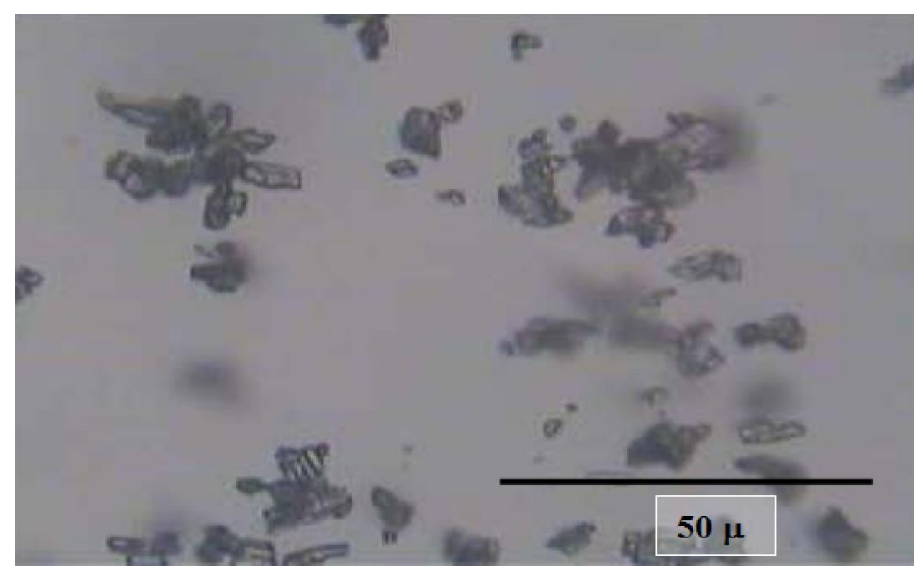

Figure 2. SEM image of old polymorph of desvenlafaxine

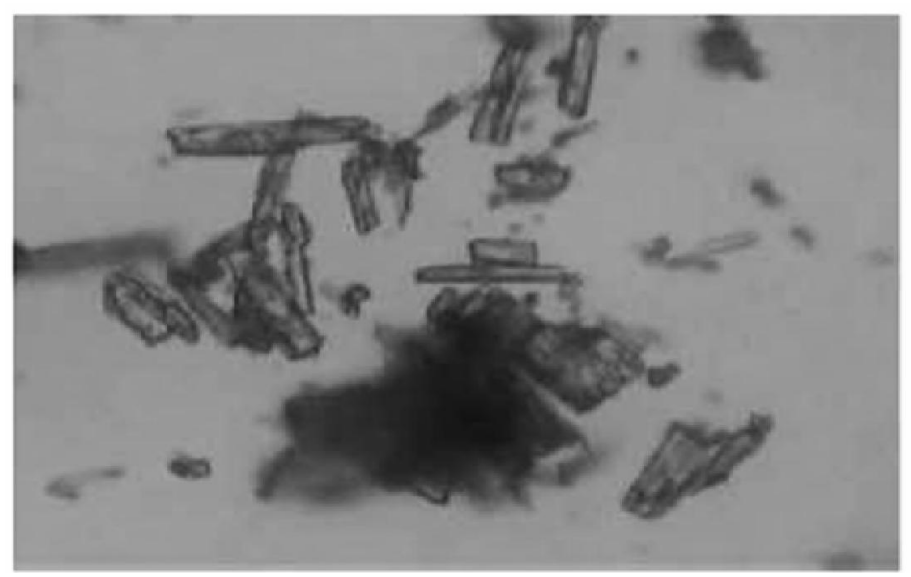

Figure 3. SEM image of new polymorph of desvenlafaxine

Between wave numbers $400 \mathrm{~cm}^{-1}$ to $4000 \mathrm{~cm}^{-1}$, a close match in the IR vibrations with an error of $2 \mathrm{~cm}^{-1}$ has been observed. Presence of two new peaks has been noted at $3128.32 \mathrm{~cm}^{-1}$ and at $3028.03 \mathrm{~cm}^{-1}$ in the new polymorph. The peak at $764.40 \mathrm{~cm}^{-1}$ in the old polymorph is seen to shift by about $18 \mathrm{~cm}^{-1}$ on the lower wave number side in the new polymorph.

\section{HPLC chromatographic analysis}

High performance liquid chromatography (HPLC) is essentially a separation technique which is used invariably for the separation of organic molecules/ions. HPLC is also extremely useful to judge the purity of the sample. Table III shows chromatographic measurements on old and new polymorphs of desvenlafaxine. The retention peaks (retention time) and hence the area for the new polymorphs is found to be enhanced, Thus the new polymorph can be ascertained to be more pure than the old polymorph.

In a nutshell the new polymorph is found to be much more ordered (elongated needle shaped structures) as against aggregates found in the case of the old polymorph. The new polymorph is thus more pure (crystalline) and exhibits enhanced solubility as compared to the old polymorph. The purity of the new polymorph remains supported further by the HPLC measurements. The XRD structure is found to be in close agreement with that reported by Venu et al., 2008. XRD studies on new polymorph of desvenlafaxine show better phase formation (with enhanced intensity peaks) as compared to that of the old polymorph. The new polymorph is also found to have greater thermal stability then the old 
Table II. Comparative IR chart showing peaks for old and new polymorphs

\begin{tabular}{|c|c|c|}
\hline Peak No. & Old polymorph $\left(\mathrm{cm}^{-1}\right)$ & New polymorph $\left(\mathrm{cm}^{-1}\right)$ \\
\hline 1 & 451.31 & $*$ \\
\hline 2 & 518.89 & 516.89 \\
\hline 3 & 545.82 & 545.02 \\
\hline 4 & 764.40 & 746.40 \\
\hline 5 & 806.19 & 806.19 \\
\hline 6 & 837.05 & 837.05 \\
\hline 7 & 952.77 & 952.77 \\
\hline 8 & 1033.77 & 1029.92 \\
\hline 9 & 1095.49 & * \\
\hline 10 & 1153.35 & 1153.35 \\
\hline 11 & 1184.21 & 1184.21 \\
\hline 12 & 1247.86 & 1247.86 \\
\hline 13 & $*$ & 1269.07 \\
\hline 14 & 1290.29 & * \\
\hline 15 & 1352.01 & 1350.08 \\
\hline 16 & 1396.37 & 1396.37 \\
\hline 17 & 1415.65 & 1415.65 \\
\hline 18 & 1450.37 & 1452.30 \\
\hline 19 & 1515.94 & * \\
\hline 20 & 1602.74 & 1602.74 \\
\hline 21 & 1645.17 & 1647.10 \\
\hline 22 & 1878.54 & 1876.61 \\
\hline 23 & 2150.48 & 2152.41 \\
\hline 24 & 2335.64 & * \\
\hline 25 & 2441.71 & 2339.49 \\
\hline 26 & 2530.43 & 2530.43 \\
\hline 27 & 2636.71 & 2636.71 \\
\hline 28 & 2715.79 & 1715.59 \\
\hline 29 & 2761.87 & 2759.95 \\
\hline 30 & 2848.67 & 2848.67 \\
\hline 31 & 2933.53 & 2933.53 \\
\hline 32 & 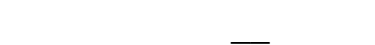 & 3028.03 \\
\hline 33 & 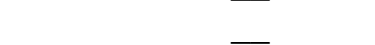 & 3128.20 \\
\hline 34 & $3166 . \overline{90}$ & 3166.90 \\
\hline 35 & 3458.13 & 3456.20 \\
\hline
\end{tabular}

polymorph which is clearly indicated by $5^{\circ} \mathrm{C}$ rise in melting point of the new polymorph. Majority of the vibrational (FTIR) peaks are found to be present in both old as well as new polymorphs confirming the presence of fundamental groups of desvenlafaxine.

\section{CONCLUSION}

The new polymorphic form of desvenlafaxine has been synthesized. More ordered, needle like elongated crystallites have been obtained for the new polymorphs as evidenced by SEM measurements. The detailed processes for making different polymorphic forms have been outlined and the relevant reaction mechanisms have been proposed. The new polymorph has been compared with the old polymorph using XRD, DSC, FTIR, SEM and HPLC techniques. Three prominent reflections with higher intensities lying at 20.3, 13.1 and 15.8 have been noted in the XRD measurements for the new polymorph. Higher 
Table III. Chromatographic measurements of old and new polymorph of Desvenlafaxine

\begin{tabular}{lccc}
\hline & RT & \% Area & RT Ratio \\
\hline Desvenlafaxine (Old- Peak 2) & 23.500 & 0.09 & 1.265 \\
Desvenlafaxine (New -Peak 2) & 23.860 & 0.04 & 1.273 \\
Desvenlafaxine (Old) & 18.577 & 99.91 & - \\
Desvenlafaxine (New) & 18.737 & 99.96 & - \\
\hline
\end{tabular}

intensity (XRD) peaks for the new polymorph indicates good crystalline quality. FTIR spectra shows close match between the polymorphs. DSC measurements show enhanced thermal stability for the new polymorph. Higher purity is indicated by the HPLC studies.

\section{ACKNOWLEDGEMENT}

We are thankful to the Principal of CK T College, Mumbai for providing the support for this research work.

\section{REFERENCES}

Abirami G., Logesh R., Vetrichelvan T., 2011, Spectrophotometric method for estimation of desvenlafaxine succinate in tablet dosage form, Res. Pharm. Biol. Che. Sci., 2, 721-729

Archer, DF., Dupont, C.M., Constantine, GD., Pickar, JH., Oliver S., 2009, Desvenlafaxine for the treatment of vasomotor symptoms associated with menopause: a double-blind, randomized, placebo-controlled trial of efficacy and safety, Am. J. Obestet. Gynecol., 200, e1238

Brittain HG., 2009. Polymorphism and Solvtomorphism, J. Pharm. Sc., 98, 16171642

De Martinis NA., Yeung PP., Entsuah R., Manley AL., 2007, A double-blind, placebo-controlled study of the efficacy and safety of desvenlafaxine succinate in the treatment of major depressive disorder, J. Clin. Psychiatry., 68, 677-88
Giron D., 2002. Applications of thermal analysis and coupled techniques in pharmaceutical Industry, J. Therm. Anal. Calorim., 68, 335-357

Kornstein SG., Jiang Q., Reddy S., Musgnung, JJ., Guico-Pabia CJ., 2010, Short-term efficacy and safety of desvenlafaxine in a randomized, placebo-controlled study of perimenopausal and postmenopausal women with major depressive disorder, J. Clin. Psychiatry, 71, 1088-1096

Liebowitz MR., Tourian KA., 2010, Efficacy, Safety, and Tolerability of Desvenlafaxine $50 \mathrm{mg} / \mathrm{d}$ for the Treatment of Major Depressive Disorder: A Systematic Review of Clinical Trials, J. Clin. Psychiatry, 12, 3-4

Lourenco MT., Kennedy SH., 2009, Desvenlafaxine in treatment of major depressive disorder, Neuropsychiatr. Dis. Treat., 127-136

Retrieved

http:/www.faqs.org/patents/app/20100 121108

Umand, E. M., Falconieri, L., 2012, Treatment options for vasomotor symptoms in menopause: focus on desvenlafaxine, Int.J. Womens. Health., 305-319

Venu N., Sreekanth BR., Ram T., Devarakonda S., 2008. Desvenlafaxine succinate Monohydrate, Acta. Crystallogr. C., 64, 290- 292 